The Astrophysical Journal, 485:680-688, 1997 August 20

(C) 1997. The American Astronomical Society. All rights reserved. Printed in U.S.A.

\title{
MAGNETOHYDRODYNAMIC TURBULENCE REVISITED
}

\author{
P. GOLDREICH ${ }^{1}$ AND S. SRIDHAR ${ }^{2}$ \\ Received 1995 September 5 ; accepted 1997 March 25
}

\begin{abstract}
In 1965, Kraichnan proposed that MHD turbulence occurs as a result of collisions between oppositely directed Alfvén wave packets. Recent work has generated some controversy over the nature of nonlinear couplings between colliding Alfvén waves. We find that the resolution to much of the confusion lies in the existence of a new type of turbulence, intermediate turbulence, in which the cascade of energy in the inertial range exhibits properties intermediate between those of weak and strong turbulent cascades. Some properties of intermediate MHD turbulence are the following: (1) in common with weak turbulent cascades, wave packets belonging to the inertial range are long-lived; (2) however, components of the strain tensor are so large that, similar to the situation in strong turbulence, perturbation theory is not applicable; (3) the breakdown of perturbation theory results from the divergence of neighboring field lines due to wave packets whose perturbations in velocity and magnetic fields are localized, but whose perturbations in displacement are not; (4) three-wave interactions dominate individual collisions between wave packets, but interactions of all orders $n \geq 3$ make comparable contributions to the intermediate turbulent energy cascade; (5) successive collisions are correlated since wave packets are distorted as they follow diverging field lines; (6) in common with the weak MHD cascade, there is no parallel cascade of energy, and the cascade to small perpendicular scales strengthens as it reaches higher wavenumbers; (7) for an appropriate weak excitation, there is a natural progression from a weak, through an intermediate, to a strong cascade.
\end{abstract}

Subject headings: ISM: general — MHD — turbulence

\section{INTRODUCTION}

There appears to be some consensus regarding the notion that turbulence in the ionized interstellar medium is of magnetohydrodynamic origin. However, even three decades after Iroshnikov (1963) and Kraichnan (1965) first, independently, presented their ideas on MHD turbulence (in an incompressible, highly conducting fluid), there is much debate on what this theory really is. Recent controversy has centered on the existence, or otherwise, of certain nonlinear interactions among Alfvén waves. Sridhar \& Goldreich (1994, hereafter SG) have argued that there are no resonant three-wave interactions in weak MHD turbulence. They also asserted that the Iroshnikov-Kraichnan (IK) theory is incorrect and constructed a theory of weak MHD turbulence based on resonant four-wave interactions; in this theory, nonlinear interactions strengthen on small spatial scales, resulting ultimately in a strong cascade proposed by Goldreich \& Sridhar (1995). However, Montgomery \& Matthaeus (1995) have maintained that resonant threewave interactions are nonempty, holding these responsible for the anisotropic cascade seen in the numerical simulations of Shebalin, Matthaeus, \& Montgomery (1983). Moreover, Ng \& Bhattacharjee (1996, hereafter NB) have recently presented calculations that show that smallamplitude wave packets do interact via three waves, so long as the $k_{z}=0(\hat{z}$ is the direction of the local, mean magnetic field) Fourier components of velocity and magnetic field perturbations are nonzero. The present investigation is an analysis and resolution of this controversy.

We consider magnetic turbulence in an incompressible fluid, although we simply call it MHD turbulence. When

\footnotetext{
${ }^{1}$ Division of Geological and Planetary Sciences, California Institute of Technology, Pasadena, CA 91125; pmg@nicholas.caltech.edu.

${ }^{2}$ Inter-University Centre for Astronomy and Astrophysics, Post Bag 4, Ganeshkhind, Pune 411 007, India; sridhar@iucaa.ernet.in.
}

the density and transport properties of the fluid are constant, the equations of (incompressible) MHD read

$$
\begin{gathered}
\partial_{t} \boldsymbol{b}=\boldsymbol{\nabla} \times(\boldsymbol{v} \times \boldsymbol{b})+\kappa \nabla^{2} \boldsymbol{b}, \\
\partial_{t} \boldsymbol{v}=-(\boldsymbol{v} \cdot \nabla) \boldsymbol{v}+(\boldsymbol{b} \cdot \boldsymbol{\nabla}) \boldsymbol{b}-\nabla p+\gamma \nabla^{2} \boldsymbol{v}, \\
\nabla \cdot \boldsymbol{v}=\boldsymbol{\nabla} \cdot \boldsymbol{b}=0,
\end{gathered}
$$

where $v$ is the velocity, $b=B /(4 \pi \rho)^{1 / 2}$ is the magnetic field in velocity units, and $p$ is the ratio of total pressure to the density. The dissipation provided by $\kappa$ and $\gamma$ is important only on small spatial scales, so we ignore the dissipative terms. The homogeneous state, $v_{0}=0, B_{0}=B_{0} \hat{z}$, is a stable, static solution of equations (1a)-(1c). Shear Alfvén waves and pseudo-Alfvén waves are the two kinds of linear perturbations about this equilibrium, the latter being the incompressible limit of the slow magnetosonic wave. Both kinds of waves have the same dispersion relation, namely, $\omega=v_{\mathrm{A}}\left|k_{z}\right|$, where $v_{\mathrm{A}}=B_{0} /(4 \pi \rho)^{1 / 2}$ is called the Alfvén speed. The perturbed velocity and magnetic fields are related by $\delta v= \pm \delta b$, where the upper/lower signs correspond to waves traveling antiparallel/parallel to $\boldsymbol{B}_{0}$ (with $k_{z}<0$ and $k_{z}>0$, respectively). Equations (1a)-(1c), with $\kappa=\gamma=0$, possess the remarkable property of allowing for nonlinear generalizations of the linear Alfvén waves. Mutual cancellation of nonlinear terms permits the following wide class of exact solutions: if $\delta v(x)=-\delta b(x)$ at some instant of time, $t=0$, it can be checked that $\delta v(x, y, z$ $\left.-v_{\mathrm{A}} t\right)=-\delta \boldsymbol{b}\left(x, y, z-v_{\mathrm{A}} t\right)$ for all time, irrespective of the functional form of $\delta v(x)$ (see Parker 1979). This nonlinear solution describes a wave packet of arbitrary form traveling nondispersively in the direction of $\boldsymbol{B}_{0}$. Similarly, we can also construct another class of nonlinear solutions, with $\delta \boldsymbol{v}=\delta \boldsymbol{b}$, that travels nondispersively in a direction opposite to $\boldsymbol{B}_{0}$. Both types of nonlinear solutions are stable, and the dynamics is simple so long as there is no spatial overlap ("collisions") between oppositely moving wave packets. 
Let us consider a situation that is less restrictive than perturbations about a static equilibrium (a uniform magnetic field). We imagine that turbulent motions on a large scale are set up in the fluid by stirring it in a random but statistically steady fashion. These create large-scale, disordered velocity and magnetic fields. Kinetic and magnetic energies will cascade to smaller spatial scales. ${ }^{3}$ The central problem of MHD turbulence is to determine the statistical steady state amplitudes of the fluctuations in $\boldsymbol{v}$ and $\boldsymbol{b}$, on intermediate spatial scales - the so-called inertial-range power spectrum. Mathematically speaking, this cascade through spatial scales should emerge from the effect of the nonlinear terms in equations (1a)-(1c). Physically, it is common to speak of interactions between "eddies" (see Frisch 1995). Do interactions between eddies of dissimilar sizes make significant contributions to the form of the cascade? For hydrodynamic turbulence, the answer seems to be that no, the dominant interactions are between eddies of similar spatial scales. The reason for such a locality in interactions between eddies is that the sweeping due to the velocity field of a large-sized eddy (on a smaller eddy) may be transformed away by a local Galilean transformation. Kraichnan noted that, for MHD turbulence, such a transformation has no effect at all on the magnetic field of the large eddy; the magnetic field of a large eddy acts upon smaller eddies in much the same manner as the mean magnetic field does on Alfvén wave packets. Hence MHD turbulence in an incompressible fluid should reduce to turbulence of interacting Alfvén wave packets. We recall from the previous paragraph that finite-amplitude wave packets that travel in the positive/negative $z$-directions do so without change in form, so long as oppositely directed packets do not overlap. Kraichnan realized that the cascade of energy in MHD turbulence occurs as a result of collisions between oppositely directed Alfvén wave packets.

To derive the results of the IK theory, consider a statistically steady, isotropic excitation of amplitude $v_{l} \ll v_{\mathrm{A}}$, on outer scale $l$, of the static equilibrium mentioned earlier. Alfvén waves have $\delta v \sim \delta b$, so that $v_{l}$ is the amplitude of excitation of both velocity and magnetic fields. The resulting fluctuations may, at any time, be decomposed into Alfvén wave packets with scales $\lambda \lesssim l$ traveling in the positive and negative $z$-directions. Iroshnikov and Kraichnan assumed that the energy transfer is local and isotropic in $\boldsymbol{k}$-space. Collisions between oppositely directed wave packets occur over times of order $\omega_{k}^{-1} \sim\left(v_{\mathrm{A}} k\right)^{-1}$, and these create small distortions. During one collision, each wave packet suffers a fractional perturbation

$$
\frac{\delta v_{\lambda}}{v_{\lambda}} \sim \frac{d v_{\lambda}}{d t}\left(v_{\mathrm{A}} k v_{\lambda}\right)^{-1} \sim \frac{v_{\lambda}}{v_{\mathrm{A}}} \ll 1 .
$$

During successive collisions, these perturbations add with random phases. The number of collisions for the fractional perturbations to build up to order unity is

$$
N_{\lambda} \sim\left(\frac{v_{\lambda}}{\delta v_{\lambda}}\right)^{2} \sim\left(\frac{v_{\mathrm{A}}}{v_{\lambda}}\right)^{2} .
$$

The energy cascade rate is $\epsilon \sim v_{\lambda}^{2} / t_{\lambda}^{2}$, where the cascade time is given by $t_{\lambda} \sim N_{\lambda} /\left(v_{\mathrm{A}} k\right) \sim v_{\mathrm{A}} /\left(k v_{\lambda}^{2}\right)$. The three-dimensional energy spectrum, $E(k)$, is related to the velocity fluctuation

\footnotetext{
${ }^{3}$ On scales small enough for the $\kappa \nabla^{2} \boldsymbol{b}$ and the $\gamma \nabla^{2} v$ terms to be important, kinetic and magnetic energies will dissipate into heat.
}

by $v_{\lambda}^{2} \sim k^{3} E(k)$. Using Kolmogorov's hypothesis of the scale independence of $\epsilon$, we obtain the following scalings for the inertial range of the IK theory:

$$
\frac{v_{\lambda}}{v_{l}} \sim\left(\frac{\lambda}{l}\right)^{1 / 4}, \quad E(k) \sim \frac{v_{l}^{2}}{l^{1 / 2} k^{7 / 2}} .
$$

With these scalings

$$
N_{\lambda} \sim\left(\frac{v_{\mathrm{A}}}{v_{l}}\right)^{2}\left(\frac{l}{\lambda}\right)^{1 / 2},
$$

so the cascade weakens as $\lambda$ decreases.

\section{INTERMEDIATE TURBULENCE}

\subsection{A New Cascade Based on Three-Wave Interactions}

SG argued that the IK theory, although seemingly plausible, was basically incorrect. Here we discuss the reason for the failure of this theory and propose a new cascade based on three-wave interactions. Let us begin by listing three key features of the IK theory:

1. Wave packets of size $\lambda$ live for $N_{\lambda}$ wave periods; large values of $N_{\lambda}$ correspond to weak interactions between wave packets of size $\lambda$. From equations (3) and (4), it may be verified that $N_{\lambda} \propto 1 / \lambda^{1 / 2}$, so that the cascade weakens as it progresses into the inertial range.

2. From equation (2), the fractional perturbation suffered by a wave packet during one collision is $\sim\left(v_{\lambda} / v_{\mathrm{A}}\right) \ll 1$; in the IK theory, interactions between wave packets are described by the lowest order nonlinear terms.

3. Isotropy is assumed in the derivation of the IK theory.

The derivation of this theory is essentially heuristic, roughly as given above in equations (2)-(4). We note this so that it is clear to the reader that there is not a more rigorous version of the "theory" that we happened not to mention. Together, features 1 and 2 imply that the "elementary interactions" between Alfvén waves must satisfy the threewave resonance conditions

$$
\boldsymbol{k}_{1}+\boldsymbol{k}_{2}=\boldsymbol{k}_{3}, \quad \omega_{1}+\omega_{2}=\omega_{3},
$$

where $\omega_{k}=v_{\mathrm{A}}\left|k_{z}\right|$. Shebalin et al. (1983) noted that the only nontrivial solutions of equations (6) require that one of either $k_{1, z}$ or $k_{2, z}$ must be zero. This implies that waves with values of $k_{z}$ not present initially cannot be created during collisions between oppositely directed wave packets. As they point out, there is no parallel (i.e., along $k_{z}$ ) cascade of energy. Thus the turbulence must be anisotropic, and energy should cascade to large $k_{\perp}$.

In what follows, we derive an anisotropic version of IK theory. As before, we imagine that the system is stirred in a statistically steady and isotropic fashion such that $v_{l} \ll v_{\mathrm{A}}$ on outer scale $l$. The absence of a parallel cascade implies that wave packets belonging to the inertial range have parallel scales $l$ and perpendicular scales $\lambda \ll l$. We estimate the spectrum of the anisotropic cascade by modifying the arguments of equations (2)-(4) so as to keep track of parallel and perpendicular scales. In one collision between two such oppositely directed wave packets, the fractional perturbation is given by

$$
\frac{\delta v_{\lambda}}{v_{\lambda}} \sim \frac{d v_{\lambda}}{d t}\left(v_{\mathrm{A}} k_{z} v_{\lambda}\right)^{-1} \sim \frac{l v_{\lambda}}{\lambda v_{\mathrm{A}}} \ll 1 .
$$


Adding up the perturbations due to successive collisions with random phases, the number of collisions over which the perturbations grow to order unity is

$$
N_{\lambda} \sim\left(\frac{v_{\lambda}}{\delta v_{\lambda}}\right)^{2} \sim\left(\frac{\lambda v_{\mathrm{A}}}{l v_{\lambda}}\right)^{2} .
$$

Making use of the steady state relation

$$
\frac{v_{\lambda}^{2}}{t_{\lambda}} \sim \frac{v_{l}^{2}}{t_{l}} \sim \epsilon
$$

("Kolmogorov's hypothesis"), we obtain scaling relations for the velocity fluctuations, as well as the threedimensional energy spectrum of the anisotropic, three-wave cascade:

$$
\frac{v_{\lambda}}{v_{l}} \sim\left(\frac{\lambda}{l}\right)^{1 / 2}, \quad E\left(k_{z}, k_{\perp}\right) \sim \frac{v_{l}^{2}}{k_{\perp}^{3}} .
$$

It follows that

$$
N_{\lambda} \sim\left(\frac{v_{\mathrm{A}}}{v_{l}}\right)^{2} \frac{\lambda}{l},
$$

so nonlinearity increases along the inertial range of the new cascade.

The anisotropic cascade differs from the original IK cascade in (1) being anisotropic, (2) having a different spectrum, and (3) strengthening at high wavenumber. This final difference has a profound consequence. It implies that the anisotropic cascade has a limited inertial range, thereby diminishing its applicability in astronomical contexts, where the excitation at the outer scale is likely to be quite strong.

It turns out that this anisotropic cascade is an example of a new type of turbulence, which we call intermediate turbulence, because it has properties intermediate between those of weak and strong turbulence. In particular, intermediate turbulence does not submit to perturbation theory.

\subsection{The Failure of Perturbation Theory in Intermediate Turbulence}

\subsubsection{Field-Line Geometry}

To lowest order in perturbation theory, wave packets move along field lines. Thus the breakdown of perturbation theory may be understood physically by studying the geometry of the divergence of a bundle of field lines. Assume that the mean field lies along the $z$-axis. Consider wave packets localized in velocity and magnetic field perturbations, but not in displacement, having longitudinal scale $l$ and transverse scale $\lambda$ with $\lambda / l<1$. Let us require that the velocity fluctuation, $v_{\lambda}$, is small enough,

$$
\chi \equiv \frac{l v_{\lambda}}{\lambda v_{\mathrm{A}}} \ll 1,
$$

so that $N_{\lambda} \gg 1$ (cf. eq. [8]). The rms differential inclination of the local field across scale $\lambda$ is $\theta_{\lambda} \sim v_{\lambda} / v_{\mathrm{A}}$. It is correlated over distances $l$ and $\lambda$ parallel and orthogonal to $z$.

Let us focus on a pair of neighboring field lines separated by $\lambda$ at $z=0$. The expectation value of the separation between these field lines, $\Delta$, varies such that

$$
\Delta^{2} \sim \lambda^{2}+\theta_{\lambda}^{2} l|z|
$$

for $|z| \gg l$. The distance along $z$ over which $|\Delta|$ increases by a factor of order unity from its initial value of $\lambda$ at $z=0$ is

$$
L_{*} \sim\left(\frac{\lambda v_{\mathrm{A}}}{l v_{\lambda}}\right)^{2} l \sim \frac{l}{\chi^{2}} ;
$$

$L_{*}$ is a function of $\lambda$. The significance of $L_{*}$ in intermediate turbulence follows because turbulence involves the transfer of energy across scales. If $\chi \ll 1$, single interactions between wave packets result in small perturbations. Cascading of energy requires of order

$$
N_{\lambda} \sim \frac{1}{\chi^{2}} \sim \frac{L_{*}}{l} \gg 1
$$

such interactions.

\subsubsection{Nonlinear Interactions in Intermediate Turbulence}

We have deduced the form of the spectrum of intermediate MHD turbulence from scaling arguments based on three-wave couplings. Moreover, these interactions are weak in the sense that $N_{\lambda} \gg 1$. This might suggest that three-wave interactions dominate those of higher order and that a rigorous derivation of the steady state cascade might result from truncation at this order. Unfortunately, this is incorrect; interactions of all orders have similar strengths.

Consider the distortion suffered during a single collision between oppositely directly wave packets of similar strength with perpendicular and parallel dimensions $\lambda \lesssim l$ and $l$. We assume that $l v_{\lambda} \ll \lambda v_{\mathrm{A}}$. Contributions from interactions involving $n$ waves may be written as

$$
\frac{\delta^{n} v_{\lambda}}{v_{\lambda}} \sim\left(\frac{l v_{\lambda}}{\lambda v_{\mathrm{A}}}\right)^{n-2} .
$$

Clearly, three-wave interactions dominate those of higher order for individual collisions.

Next we consider the cumulative distortion due to $n$-wave interactions as a wave packet travels a distance $l \ll$ $z \ll L_{*}$. We can picture the distortion as arising from the shearing of the packet as it follows the differential wandering of neighboring field lines. ${ }^{4}$ The net displacement of individual field lines over distance $z$ defines a vector field whose shear tensor transforms the packet's shape. ${ }^{5}$ For $l \ll z \ll L_{*}$ this transformation is close to the identity, so it may be expanded in a Taylor series. The expansion parameter is $\left(z / L_{*}\right)^{1 / 2}$, the dimensionless measure of fractional spreading over distance $z$ of a bundle of field lines whose crosssectional radius at $z=0$ is $\lambda$. Terms of order $n-2$ in $v_{\lambda}$ correspond to $n$-wave interactions. ${ }^{6}$ These terms have the form

$$
\frac{\delta^{n} v_{\lambda}}{v_{\lambda}} \sim\left(\frac{l v_{\lambda}}{\lambda v_{\mathrm{A}}}\right)^{n-2}\left(\frac{z}{l}\right)^{(n-2) / 2} \sim\left(\frac{z}{L_{*}}\right)^{(n-2) / 2} .
$$

A notable feature of equation (17) is that contributions from higher order $(n \geq 4)$ interactions carry extra factors of $(z / l)^{1 / 2}$. By "extra factors," we mean those beyond the single factor $(z / l)^{1 / 2}$ expected to arise from the addition of a sequence of independent interactions between pairs of wave

\footnotetext{
${ }^{4}$ A uniform displacement of a wave packet does not contribute to the energy cascade.

${ }^{5}$ This transformation is subject to the constraints of fluid incompressibility and magnetic flux freezing.

${ }^{6}$ Since wave packets follow field lines only to lowest order, nonkinematic terms appear at orders $n \geq 4$. Those of $n=4$ are given in $\S$ 3.3.2.
} 
packets. These factors reveal an interdependence among collisions associated with the nonlocalized field-line displacements of the wave packets. ${ }^{7}$

Intermediate turbulence is nonperturbative because distortions of all orders become large as $z \rightarrow L_{*}$. This is as expected, because the cascade time across scale $\lambda$ is $t_{\lambda} \sim$ $L_{*} / v_{\mathrm{A}}$. It implies that $n$-wave interactions of all orders $n \geq 3$ make comparable contributions to the energy cascade.

\subsubsection{Lagrangian Perturbation Theory}

A fluid element whose Lagrangian coordinate is $a$ has Eulerian location $x$, at time $t$, given by

$$
\boldsymbol{x}=\boldsymbol{a}+\boldsymbol{\xi}(\boldsymbol{a}, t),
$$

where $\xi(\boldsymbol{a}, t)$ is the displacement field. Velocity and magnetic field perturbations at the Eulerian location are defined in terms of the displacement vector by

$$
v(x, t)=\frac{\partial \xi}{\partial t}(a, t), \quad b(x, t)=v_{\mathrm{A}} \frac{\partial \xi}{\partial a_{\|}}(a, t) .
$$

SG employed a formulation of the MHD action due to Newcomb (1962) and developed a Lagrangian perturbation theory for weak MHD turbulence. The Lagrangian is a functional of the strain tensor field, in other words, the gradient of the displacement vector field. Expansion of the Lagrangian density in powers of the strain tensor yields terms of second order, $n=2$, and then fourth and higher orders, $n \geq 4$. The absence of third-order terms signifies that wave packets follow field lines to lowest nonlinear order. The absence of Lagrangian perturbations based on threewave interactions implies that Eulerian perturbations due to three-wave interactions are purely kinematic. Kinematic contributions to Eulerian perturbations are also present at each higher order, $n \geq 4$. But these are augmented by dynamic perturbations arising from order $n \geq 4$ terms in the expansion of the Lagrangian.

As before, we consider small-amplitude, $\left(l v_{\lambda} / \lambda v_{l}\right) \ll 1$, wave packets localized in $v$ and $b$ but not in $\xi$. Roughly speaking, convergence of the perturbative expansion requires the components of the strain tensor to be smaller than unity. ${ }^{8}$ An ensemble of independent wave packets generates an energy spectrum that is flat for $k_{z} l \lesssim 1$. Since $\omega=$ $v_{\mathrm{A}}\left|k_{z}\right|$, the power spectrum of the displacement vector field varies as $k_{z}^{-2}$ for $k_{z} l \ll 1$. The same behavior characterizes the power spectra of some of the components of the strain tensor. It implies that these components diverge. The divergence is the mathematical expression of the spreading of field-line bundles described in $\S 2.2 .1$. In this light, the failure of perturbation theory is seen to be generic, and not just a consequence of an unfortunate choice of perturbation variable.

Next we investigate the effect of cutting off the energy spectrum below $k_{z} L \sim 1$, where $L \gtrsim l$. The most strongly divergent components of $\xi^{i j}$ have power spectra given by

$$
\left|\tilde{\xi}^{i j}(\boldsymbol{k})\right|^{2} \sim\left(\frac{v_{\lambda}}{v_{\mathrm{A}}}\right)^{2} \frac{l}{k_{z}^{2}} .
$$

\footnotetext{
${ }^{7}$ These extra factors of $(z / l)^{1 / 2}$ are absent in the corresponding formula for wave packets that are localized in displacement.

${ }^{8}$ From this point on it is best to proceed in Fourier space, since the breakdown of perturbation theory is closely related to the behavior of the energy spectrum (i.e., velocity, or magnetic field power spectra) at small $k_{z}$.
}

Multiplying equation (20) by $k_{\perp}^{2}$ and integrating from $k_{z} \sim$ $L^{-1}$ to $k_{z} \sim l^{-1}$, we obtain the average value of $\left|\xi^{i j}\right|^{2}$ due to power in this wavenumber interval,

$$
\overline{\left|\xi^{i j}\right|^{2}} \sim \lambda^{-2} \int_{L^{-1}}^{l^{-1}} d k_{z}\left|\tilde{\xi}^{i j}(\boldsymbol{k})\right|^{2} \sim\left(\frac{l v_{\lambda}}{\lambda v_{\mathrm{A}}}\right)^{2} \frac{L}{l} .
$$

Thus

$$
\overline{\left|\xi^{i j}\right|^{2}} \sim L / L_{*},
$$

where $L_{*}$ is defined in equation (14). Once again we see the crucial role played by $L_{*}$; the perturbative expansion converges if the energy spectrum is cut off below $k_{z} L_{*} \sim 1$.

The absence of third-order terms in the expansion of the Lagrangian signifies the absence of resonant three-wave interactions in perturbative MHD turbulence. Weak MHD turbulence based on four-wave interactions is discussed in $\S 3.2$.

\section{ON WEAK AND INTERMEDIATE TURBULENCE}

\subsection{Types of Turbulence}

We have discussed, at some length, the intermediate cascade. It is time to state in a precise manner the properties that characterize the three kinds of turbulence. Let us begin with a definition. Weak turbulence is characterized by the following properties: ${ }^{9}$

1. Nonlinear interactions among an ensemble of waves that are weak, in the sense that the fractional change in wave amplitude during each wave period is small.

2. The existence of a convergent perturbative expansion for the nonlinear interactions. Typically the small parameter is the fractional change in wave amplitude during a wave period.

When these two conditions are satisfied, a formal theory of resonant wave interactions may be derived. For the theory to be nonempty, either the three-wave or four-wave resonance relations must possess nontrivial solutions. Power spectra of cascades arise as stationary solutions of the kinetic equation describing modal energy transfer.

Specifically, for MHD turbulence, we find the following:

a) The turbulent cascade based on four-wave interactions, derived in SG, is the unique weak cascade that satisfies both conditions 1 and 2 above. Moreover, this cascade is realizable; it could in principle be set up experimentally. While three-wave interactions do not vanish, they are nonresonant and do not transfer energy among different waves.

b) The critically balanced cascade described in Goldreich $\&$ Sridhar (1995) is an example of strong turbulence. It violates both conditions 1 and 2 . The interaction time is of the order of the wave period. Interactions of all orders have comparable strengths; there is no valid perturbative expansion.

c) There is a third type of MHD turbulence that satisfies condition 1 but not condition 2. It is an example of what we have called intermediate turbulence. This turbulence exhibits weak interactions, but strains in the fluid are so strong that perturbation theory diverges. The three-wave interactions are included, but not dominant; it turns out that inter-

\footnotetext{
${ }^{9}$ For a standard discussion of these points, see the introduction to Zakharov, L’vov, \& Falkovich (1992).
} 
actions of all orders contribute equally weakly. The inertialrange spectrum of intermediate MHD turbulence is given, for the first time, in equation (10) of this paper.

\subsection{Weak Alfvénic Turbulence}

Having discussed intermediate turbulence in some detail, we provide a brief outline of the theory of weak MHD turbulence that SG constructed using resonant four-wave interactions. By studying the resonant terms of the fourthorder Lagrangian, SG derived a formal kinetic equation for the evolution of energies (more precisely, "wave action") in different modes and proved that a cascade of energy indeed emerges as a stationary solution. The elementary interactions involve scattering of two waves that respect the following conservation laws:

$$
\boldsymbol{k}_{1}+\boldsymbol{k}_{2}=\boldsymbol{k}_{3}+\boldsymbol{k}_{4}, \quad \omega_{1}+\omega_{2}=\omega_{3}+\omega_{4} .
$$

Using $\omega_{k}=v_{\mathrm{A}}\left|k_{z}\right|$ and the $z$-component of the equation involving the $k$ 's, SG proved that $k_{1, z}=k_{3, z}>0$ and $k_{2, z}=k_{4, z}<0$. Of course, the symmetry of equation (23) allows us to flip the signs of all the $k_{z}$ 's, or permute indices 3 and 4 ; the important point is that the scattering process described by equation (23) leaves the $k_{z}$-components unaltered. This implies that waves with values of $k_{z}$ not present in the external stirring cannot be created by resonant fourwave interactions.

In addition to developing a formal theory, SG also provided a heuristic derivation of the weak four-wave cascade for shear Alfvén waves. Here we note the main properties of the weak cascade of shear Alfvén waves:

1. As discussed above, there is no transfer of energy to small spatial scales in the $z$-direction; the energy cascade in $\boldsymbol{k}$-space occurs only along $\boldsymbol{k}_{\perp}$ (i.e., in directions perpendicular to the mean magnetic field).

2. The three-dimensional energy spectrum, $E$, is defined by

$$
\sum v_{\lambda}^{2}=\int E\left(k_{z}, k_{\perp}\right) \frac{d^{3} k}{8 \pi^{3}},
$$

where the sum is over wave packets of various scales. Weak turbulence relies on a convergent perturbation theory. As discussed in $\S 2.2 .3$, this requires that the spectrum, $E$, be cut off for $\left|k_{z} L_{*}\right|<1$. Moreover, weak four-wave interactions do not change $k_{z}$, which implies that $E$ may have a quite arbitrary dependence on $\left|k_{z}\right|$. This simply depends on the nature of the excitation and is not of much interest here. If $\lambda \sim k_{\perp}^{-1}$ is a perpendicular length scale belonging to the inertial range, the scalings derived by SG for the weak, four-wave cascade are

$$
\frac{v_{\lambda}}{v_{l}} \sim\left(\frac{\lambda}{l}\right)^{2 / 3}, \quad E\left(k_{z}, k_{\perp}\right) \sim \frac{v_{l}^{2}}{l^{1 / 3} k_{\perp}^{10 / 3}} .
$$

3. The number of collisions needed for the packet to lose memory of its initial state is

$$
N_{\lambda} \sim\left(\frac{\lambda v_{\mathrm{A}}}{l v_{\lambda}}\right)^{4} \sim\left(\frac{v_{\mathrm{A}}}{v_{l}}\right)^{4}\left(\frac{\lambda}{l}\right)^{4 / 3} .
$$

Note that, in common with the spectrum of intermediate turbulence (given in eq. [10]), $N_{\lambda}$ decreases as the cascade proceeds to smaller $\lambda$.

4. In their treatment of weak turbulence, SG unwittingly made the assumption that $E$ was cut off at small $\left|k_{z}\right|$. While the weak turbulence of SG is realizable (see $\S 4$ below), this feature makes it less applicable than intermediate turbulence.

\subsection{A Controversy and Its Resolution}

NB have proved, analytically, that three-wave interactions between small-amplitude wave packets are nonzero if the $k_{z}=0$ Fourier components are nonzero. On the other hand, SG have argued, using a Lagrangian perturbation theory, that three-wave interactions are absent in weak MHD turbulence. In $\S 2$, we claimed that the interactions found by NB lead to intermediate, rather than weak, turbulence. Here we bolster that claim by an explicit evaluation of three-wave and four-wave interactions in Lagrangian coordinates.

The simplest derivation employs the Lagrangian displacement vector field as the basic variable (cf. eqs. [18] and [19]). Incompressibility implies that the transformation between Lagrangian and Eulerian coordinates have unit Jacobian. Thus

$$
\begin{aligned}
J= & 1+\nabla \cdot \xi-\frac{1}{2} \nabla \xi: \nabla \xi+\frac{1}{2}(\nabla \cdot \xi)^{2}+\frac{1}{3} \xi^{i j} \xi^{j k} \xi^{k i} \\
& -\frac{1}{2}(\nabla \cdot \xi) \xi^{i j} \xi^{j i}+\frac{1}{6}(\nabla \cdot \xi)^{3},
\end{aligned}
$$

where $\nabla$ refers to derivatives with respect to Lagrangian coordinates and

$$
\nabla \xi: \nabla \xi \equiv \xi^{i j} \xi^{j i} .
$$

The displacement vector $\xi$ is split into transverse and longitudinal components $\boldsymbol{\eta}$ and $\zeta$, respectively, such that

$$
\boldsymbol{\xi}=\boldsymbol{\eta}+\zeta,
$$

with

$$
\boldsymbol{\nabla} \cdot \boldsymbol{\eta}=0 .
$$

The components of $\boldsymbol{\eta}$ are the two independent variables; $\zeta$ is obtained from equation (27). Thus

$$
\begin{gathered}
\nabla \cdot \zeta_{2}=\frac{1}{2} \nabla \boldsymbol{\eta}_{1}: \nabla \eta_{1}, \\
\nabla \cdot \zeta_{3}=\nabla \eta_{1}: \nabla \eta_{2}+\nabla \eta_{1}: \nabla \zeta_{2}-\frac{1}{3} \eta_{1}^{i j} \eta_{1}^{j k} \eta_{1}^{k i} .
\end{gathered}
$$

The first term on the right-hand side of equation (32) is included for completeness, since $\boldsymbol{\eta}_{2}=0 .{ }^{10}$

Following the development in $\S 3$ of SG, we write the Lagrangian as

$$
\mathscr{L}=\frac{\rho}{2} \int d^{3} a\left(\left|\frac{\partial \xi}{\partial t}\right|^{2}-v_{\mathrm{A}}^{2}\left|\frac{\partial \xi}{\partial a_{\|}}\right|^{2}\right) .
$$

However, we present our calculations in real space, rather than in Fourier space; the results are identical, but the realspace version turns out to be useful later. Solving equation (31) yields

$$
\zeta_{2}=-\nabla \int \frac{d^{3} a^{\prime}}{8 \pi} \frac{\nabla \boldsymbol{\eta}_{1}: \nabla \boldsymbol{\eta}_{1}}{\left|a-a^{\prime}\right|} .
$$

We now write

$$
\mathscr{L}=\mathscr{L}_{2}+\mathscr{L}_{4}
$$

\footnotetext{
${ }^{10}$ See eq. (42) and the footnote that follows it.
} 
(the third-order terms vanish), with

$$
\begin{aligned}
& \mathscr{L}_{2}=\frac{\rho}{2} \int d^{3} a\left(\left|\frac{\partial \boldsymbol{\eta}_{1}}{\partial t}\right|^{2}-v_{\mathrm{A}}^{2}\left|\frac{\partial \boldsymbol{\eta}_{1}}{\partial a_{\|}}\right|^{2}\right), \\
& \mathscr{L}_{4}=\frac{\rho}{2} \int d^{3} a\left(\left|\frac{\partial \zeta_{2}}{\partial t}\right|^{2}-v_{\mathrm{A}}^{2}\left|\frac{\partial \zeta_{2}}{\partial a_{\|}}\right|^{2}\right) .
\end{aligned}
$$

Variation of the action

$$
\mathscr{S} \equiv \int d t\left[\mathscr{L}+\int d^{3} a P(\nabla \cdot \eta)\right]
$$

with respect to $\eta$ leads to the (Euler-Lagrange) equation of motion. ${ }^{11}$

\subsubsection{Three-Wave Interactions in Lagrangian Coordinates}

In this subsection, we recover the results of the threewave interactions calculated by NB. Moreover, we demonstrate that they are purely kinematic in Lagrangian coordinates.

To lowest order, the contribution of $\mathscr{L}_{4}$ may be ignored. Using equation (36) for $\mathscr{L}_{2}$ in the variation of $\mathscr{S}$ (eq. [38]) results in the following simple, linear equation: ${ }^{12}$

$$
\left(\frac{\partial^{2}}{\partial t^{2}}-v_{\AA}^{2} \frac{\partial^{2}}{\partial a_{\|}^{2}}\right) \boldsymbol{\eta}_{1}=0
$$

whose general solution is a superposition of wave packets traveling in the positive and negative $z$-directions:

$$
\boldsymbol{\eta}_{1}=\boldsymbol{\eta}_{1}^{+}\left(\boldsymbol{a}_{\perp}, a_{\|}-v_{\mathrm{A}} t\right)+\boldsymbol{\eta}_{1}^{-}\left(\boldsymbol{a}_{\perp}, a_{\|}+v_{\mathrm{A}} t\right) .
$$

NB calculated, in Eulerian coordinates, the lowest order perturbation due to a collision between oppositely directed wave packets. It is a trivial matter to obtain this quantity by using Lagrangian perturbation theory. To do so, we transform the right-hand side of the expression for $v$, given in equation (19), into Eulerian coordinates. To first order,

$$
\boldsymbol{v}_{1}(\boldsymbol{x}, t)=\frac{\partial \boldsymbol{\eta}_{1}}{\partial t}(\boldsymbol{x}, t)
$$

is the velocity field of unperturbed wave packets, and $\boldsymbol{\eta}_{1}$ is the corresponding displacement field. To second order,

$$
\boldsymbol{v}_{2}=-\left(\boldsymbol{\eta}_{1} \cdot \nabla_{x}\right) \boldsymbol{v}_{1}+\frac{\partial \zeta_{2}}{\partial t}
$$

where the subscript $x$ refers to Eulerian coordinates. ${ }^{13}$ When we substitute equation (40) in the right-hand side of equation (42), we obtain three different types of terms; those that contain two powers of $\boldsymbol{\eta}_{1}^{+}$or $\boldsymbol{\eta}_{1}^{-}$have nothing to do with perturbations induced by collisions. Only the mixed terms describe distortions suffered by a wave packet during a collision with an oppositely directed wave packet. If

$$
\Delta \boldsymbol{\eta}_{1}^{ \pm}=\mp\left[\boldsymbol{\eta}_{1}^{ \pm}\left(\boldsymbol{x}_{\perp},+\infty\right)-\boldsymbol{\eta}_{1}^{ \pm}\left(\boldsymbol{x}_{\perp},-\infty\right)\right]
$$

is the net displacement of field lines due to the $( \pm)$ wave packets, then the asymptotic distortion due to a collision is

$$
\Delta v_{2}^{ \pm}=-\left(\Delta \eta_{1}^{\mp} \cdot \nabla_{x}\right) v_{1}^{ \pm}-\nabla_{x} \int \frac{d^{3} x^{\prime}}{4 \pi} \frac{\nabla_{x}\left(\Delta \eta_{1}^{\mp}\right): \nabla_{x} v_{1}^{ \pm}}{\left|x-x^{\prime}\right|} .
$$

\footnotetext{
${ }^{11} P$ is a Lagrange multiplier needed to ensure that $\nabla \cdot \eta=0$.

${ }^{12} \mathrm{We}$ assign first order to $\eta$ of unperturbed wave trains.

13 The absence of $\partial \boldsymbol{\eta}_{2} / \partial t$ is due to the vanishing of $\mathscr{L}_{3}$.
}

The above expression is equivalent to equations (15) and (16) of NB. The wave packet distortions expressed by equation (44) are kinematic, as described in $\S 2.2 .3$. Displacements resulting from any sequence of collisions are obtained by summing individual values. ${ }^{14}$ Note that both terms on the right-hand side of equation (44) depend on $\Delta \boldsymbol{\eta}_{1}^{ \pm}$, the net displacement of field lines, which is related to the Fourier amplitude of $\boldsymbol{\eta}_{1}^{ \pm}$with $k_{z}=0$.

\subsubsection{Four-Wave Interactions in Lagrangian Coordinates}

Our principal aim in this subsection is to demonstrate that four-wave interactions obey the distance scaling proposed in equation (17). Our secondary goals are to obtain the spectrum of weak Alfvénic turbulence from a configuration-space calculation and to recover the frequency-changing terms discovered by $\mathrm{NB}$, in full MHD. ${ }^{15}$

We have shown that the three-wave interactions of NB arise from kinematic perturbations in Eulerian coordinates. Dynamic perturbations require the interaction of at least four waves and are associated with perturbations in Lagrangian coordinates. We already possess the machinery necessary to derive these. When $\mathscr{L}_{4}$ is included in the action of equation (38), the variation with respect to $\eta_{1}$ leads to Euler-Lagrange equations, which may be thought of as adding third-order terms to the right-hand side of equation (39):

$$
\left(\frac{\partial^{2}}{\partial t^{2}}-v_{\mathrm{A}}^{2} \frac{\partial^{2}}{\partial a_{\|}^{2}}\right) \boldsymbol{\eta}_{3}=\left(\boldsymbol{\eta}_{1} \cdot \nabla\right)\left(\frac{\partial^{2}}{\partial t^{2}}-v_{\mathrm{A}}^{2} \frac{\partial^{2}}{\partial a_{\|}^{2}}\right) \zeta_{2}-\frac{\nabla \tilde{P}_{3}}{\rho},
$$

where $\widetilde{P}_{3}$ is determined by requiring that $\nabla \cdot \boldsymbol{\eta}_{3}=0$.

For simplicity, we evaluate the deformation suffered by a wave packet traveling in the positive $a_{\|}$-direction. It proves convenient to transform to coordinates

$$
\alpha=a_{\|}-v_{\mathrm{A}} t, \quad \tau=t .
$$

As given in equation (40), the unperturbed wave packets in these coordinates have the forms

$$
\boldsymbol{\eta}_{1}^{+}=\boldsymbol{\eta}_{1}^{+}\left(\boldsymbol{a}_{\perp}, \alpha\right), \quad \boldsymbol{\eta}_{1}^{-}=\boldsymbol{\eta}_{1}^{-}\left(\boldsymbol{a}_{\perp}, \alpha+2 v_{\mathrm{A}} \tau\right) .
$$

The equation of motion for $\boldsymbol{\eta}_{3}$ in the new variables, $\tau$ and $\boldsymbol{A} \equiv\left(\boldsymbol{a}_{\perp}, \alpha\right)$, reads

$$
\begin{aligned}
\frac{\partial}{\partial \tau}\left(\frac{\partial}{\partial \tau}-2 v_{\mathrm{A}} \frac{\partial}{\partial \alpha}\right) \boldsymbol{\eta}_{3}= & -\left(\boldsymbol{\eta}_{1} \cdot \nabla\right) \frac{\partial}{\partial \tau}\left(\frac{\partial}{\partial \tau}-2 v_{\mathrm{A}} \frac{\partial}{\partial \alpha}\right) \\
& \times \nabla \int \frac{d^{3} A^{\prime}}{4 \pi} \frac{\nabla \boldsymbol{\eta}_{1}^{-}: \nabla \boldsymbol{\eta}_{1}^{+}}{\left|\boldsymbol{A}-\boldsymbol{A}^{\prime}\right|}-\frac{\nabla \widetilde{P}_{3}}{\rho},
\end{aligned}
$$

where the primes indicate dummy integration variables and the choice of superscripts applied to $\eta_{1}$ on the right-hand side of the equation is dictated by the requirement that the differential operators acting on the integral do not kill it; one $\eta_{1}^{+}$and one $\eta_{1}^{-}$must appear in the integral over $d^{3} A^{\prime}$

\footnotetext{
${ }^{14} \mathrm{SG}$ missed these distortions because they studied wave packet interactions in which the wave packets were localized in $\boldsymbol{\eta}$; for these $\Delta \boldsymbol{\eta}_{1}^{ \pm}=0$, and third-order couplings vanish even in Eulerian coordinates. This is consistent with the point made in footnote 5 of SG (p. 616), that resonant coupling coefficients are independent of the variables used. Of course, for this to be true, one must be in a regime in which perturbation theory is valid, which obtains only for wave packets localized in $\boldsymbol{\eta}$.

${ }^{15}$ NB's discovery was made using reduced MHD.
} 
because the derivatives $\partial / \partial \tau$ and $\left(\partial / \partial \tau-2 v_{\mathrm{A}} \partial / \partial \alpha\right)$ annihilate $\boldsymbol{\eta}_{1}^{+}$and $\boldsymbol{\eta}_{1}^{-}$, respectively. ${ }^{16}$

Further expansion of the right-hand side of equation (48), obtained by writing $\boldsymbol{\eta}_{1}=\boldsymbol{\eta}_{1}^{+}+\boldsymbol{\eta}_{1}^{-}$, results in two terms. Rather than carry the cumbersome pressure term through the remainder of our calculation, we discard it and replace $\eta_{3}$ on the left-hand side of the equation of motion by $\tilde{\boldsymbol{\eta}}_{3}$, to remind us that its longitudinal part must be subtracted off at a later stage:

$$
\begin{gathered}
\frac{\partial}{\partial \tau}\left(\frac{\partial}{\partial \tau}-2 v_{\mathrm{A}} \frac{\partial}{\partial \alpha}\right) \tilde{\boldsymbol{\eta}}_{3}=-\left(\frac{\partial}{\partial \tau}-2 v_{\mathrm{A}} \frac{\partial}{\partial \alpha}\right)\left(\boldsymbol{\eta}_{1}^{-} \cdot \nabla\right) \\
\times \frac{\partial}{\partial \tau} \boldsymbol{\nabla} \int \frac{d^{3} A^{\prime}}{4 \pi} \frac{\nabla \boldsymbol{\eta}_{1}^{-}: \nabla \boldsymbol{\eta}_{1}^{+}}{\left|\boldsymbol{A}-\boldsymbol{A}^{\prime}\right|}-\frac{\partial}{\partial \tau}\left(\boldsymbol{\eta}_{1}^{+} \cdot \nabla\right) \\
\times\left(\frac{\partial}{\partial \tau}-2 v_{\mathrm{A}} \frac{\partial}{\partial \alpha}\right) \nabla \int \frac{d^{3} A^{\prime}}{4 \pi} \frac{\nabla \boldsymbol{\eta}_{1}^{-}: \nabla \boldsymbol{\eta}_{1}^{+}}{\left|\boldsymbol{A}-\boldsymbol{A}^{\prime}\right|} .
\end{gathered}
$$

Although we have used coordinates, $(\alpha, \tau)$, moving with the positive wave packet, we emphasize that the equation of motion (eq. [49]) remains symmetric in $\tilde{\boldsymbol{\eta}}_{3}^{+}$and $\tilde{\boldsymbol{\eta}}_{3}^{-}$, i.e., the action of the first term on a $( \pm)$ wave packet is identical to that of the second term on a $(\bar{\mp})$ wave packet. To determine the perturbations suffered by a $(+)$ wave packet, we set $\tilde{\boldsymbol{\eta}}_{3}=\tilde{\boldsymbol{\eta}}_{3}^{+}$on the left-hand side.

If the first term were the only perturbing interaction, we could peel off the operator $\left(\partial / \partial \tau-2 v_{\mathrm{A}} \partial / \partial \alpha\right)$ from both sides. Integration over time would yield

$$
\Delta \tilde{\boldsymbol{\eta}}_{3 \mathrm{a}}^{+}=-\int_{-\infty}^{+\infty} d \tau\left(\boldsymbol{\eta}_{1}^{-} \cdot \nabla\right) \boldsymbol{\nabla} \int \frac{d^{3} A^{\prime}}{4 \pi} \frac{\nabla\left(\partial \boldsymbol{\eta}_{1}^{-} / \partial \tau\right): \nabla \boldsymbol{\eta}_{1}^{+}}{\left|\boldsymbol{A}-\boldsymbol{A}^{\prime}\right|},
$$

which describes the four-wave interactions (cf. eq. [23]) that form the basis of the weak turbulence theory of SG.

The third-order Eulerian velocity perturbation consists of both kinematic and dynamic terms. An explicit expression follows from equation (19):

$$
\begin{aligned}
\boldsymbol{v}_{3}(\boldsymbol{x}, t)= & \frac{1}{2} \boldsymbol{\eta}_{1} \boldsymbol{\eta}_{1}: \nabla_{x} \nabla_{x} \frac{\partial \boldsymbol{\eta}_{1}}{\partial t}+\left(\boldsymbol{\eta}_{1} \cdot \nabla_{x}\right)\left(\boldsymbol{\eta}_{1} \cdot \nabla_{x}\right) \frac{\partial \boldsymbol{\eta}_{1}}{\partial t} \\
& -\left(\boldsymbol{\eta}_{1} \cdot \nabla_{x}\right) \frac{\partial \zeta_{2}}{\partial t}-\left(\zeta_{2} \cdot \nabla_{x}\right) \frac{\partial \boldsymbol{\eta}_{1}}{\partial t}+\frac{\partial \zeta_{3}}{\partial t}+\frac{\partial \boldsymbol{\eta}_{3}}{\partial t} .
\end{aligned}
$$

The final term is the sole dynamic entry. Each of the other terms is constructed from $\boldsymbol{\eta}_{1}$ without use of the equation of motion. In particular, $\zeta_{3}$ is obtained from equation (32).

Let us estimate the fractional distortion suffered by a positive wave packet after traveling a distance $z \gg l$. Among the plethora of kinematic terms, consider only

$$
\Delta v_{3}^{+}=\frac{1}{2} \Delta\left(\boldsymbol{\eta}_{1}^{-} \boldsymbol{\eta}_{1}^{-}\right): \nabla_{x} \nabla_{x} \frac{\partial \eta_{1}^{+}}{\partial t} .
$$

To order of magnitude, both this term and the dynamic term contribute

$$
\frac{\delta^{4} v_{\lambda}^{+}}{v_{\lambda}^{+}} \sim\left(\frac{l v_{\lambda}^{-}}{\lambda v_{\mathrm{A}}}\right)^{2} \frac{z}{l}
$$

\footnotetext{
${ }^{16}$ Proof of the second of these relations requires an integration by parts to transfer $\partial / \partial \alpha$ acting on $1 /\left|\boldsymbol{A}-\boldsymbol{A}^{\prime}\right|$ to $\partial / \partial \alpha^{\prime}$ acting on $\nabla \boldsymbol{\eta}_{1}: \nabla \boldsymbol{\eta}_{1}$.
}

the same order of magnitude as the fractional Eulerian distortion given by equation (17). ${ }^{17}$

To make contact with the weak four-wave cascade, we suppose that the velocity spectrum of the negatively directed waves is cut off at $k_{z} \sim L^{-1} \ll l^{-1}$. ${ }^{18}$ Then the distortions build up coherently over distance $L$. Over longer distances, $z \gg L$, they add with random phases, implying

$$
\frac{\delta^{4} v_{\lambda}}{v_{\lambda}} \sim\left(\frac{l v_{\lambda}}{\lambda v_{\mathrm{A}}}\right)^{2} \frac{L}{l}\left(\frac{z}{L}\right)^{1 / 2} .
$$

Cascade occurs when $\delta^{4} v_{\lambda} / v_{\lambda} \sim 1$; thus

$$
N_{\lambda} \sim\left(\frac{\lambda v_{\mathrm{A}}}{l v_{\lambda}}\right)^{4} \frac{l}{L}
$$

But for the extra factor of $l / L$, this is identical to equation (26), whereupon, provided that $N_{\lambda} \gg 1$, the spectrum of the weak four-wave cascade, given in equation (25), follows.

Next we return to investigate the second term in equation (49). Imagine that the first term was switched off. Then remove a $\partial / \partial \tau$ from both sides, leaving the left-hand side in the form $\left(\partial / \partial \tau-2 v_{\mathrm{A}} \partial / \partial \alpha\right) \tilde{\boldsymbol{\eta}}_{3 \mathbf{b}}^{+}$. We expect the action of $\partial / \partial \tau$ to be subdominant; therefore

$$
\frac{\partial \tilde{\boldsymbol{\eta}}_{3 \mathrm{~b}}^{+}}{\partial \alpha} \simeq-\left(\boldsymbol{\eta}_{1}^{+} \cdot \nabla\right) \nabla \int \frac{d^{3} A^{\prime}}{4 \pi} \frac{\nabla \boldsymbol{\eta}_{1}^{-}: \nabla\left(\partial \boldsymbol{\eta}_{1}^{+} / \partial \alpha^{\prime}\right)}{\left|\boldsymbol{A}-\boldsymbol{A}^{\prime}\right|},
$$

implying that the net change in the $(+)$ wave packet is

$$
\Delta\left(\frac{\partial \tilde{\boldsymbol{\eta}}_{\mathrm{b}}^{+}}{\partial \alpha}\right) \simeq-\left.\left(\boldsymbol{\eta}_{1}^{+} \cdot \nabla\right) \nabla \int \frac{d^{2} a_{\perp}^{\prime}}{4 \pi} \nabla \boldsymbol{\eta}_{1}^{-}\right|_{-\infty} ^{\infty}: \int d \alpha^{\prime} \frac{\nabla\left(\partial \boldsymbol{\eta}_{1}^{+} / \partial \alpha\right)}{\left|\boldsymbol{A}-\boldsymbol{A}^{\prime}\right|} .
$$

We have written equation (57) in a form that makes explicit the dependence of the perturbation on only the net displacement induced by the (-) wave packet. In other words, the perturbation induced in a wave packet is proportional to the amplitudes of the $k_{z}=0$ Fourier components of oppositely directed wave packets: these terms were first discovered by NB in the limit of reduced MHD. We may describe the interactions by the following kind of resonant four-wave process:

$$
\boldsymbol{k}_{1}=\boldsymbol{k}_{2}+\boldsymbol{k}_{3}+\boldsymbol{k}_{4}, \quad \omega_{1}=\omega_{2}+\omega_{3}+\omega_{4},
$$

wherein one wave may be induced (by an oppositely directed wave) to split into two, or two waves could equally well be induced to combine into one. Manipulating the resonance relations leads to $k_{1, z}, k_{2, z}, k_{3, z}$ positive (say), while $k_{4, z}$ approaches zero from below. Thus $\omega_{1}=\omega_{2}+\omega_{3}$ allows for changes in the frequencies of wave packets, and harmonics, as well as subharmonics, are generated by this process. These four-wave interactions require the energy spectrum of the negatively directed packets to be flat near $k_{z}=0$; thus they do not arise in weak turbulence. Are they of importance to intermediate turbulence? To this end, let us make an order-of-magnitude estimate of the pertur-

\footnotetext{
${ }^{17}$ We draw particular attention to the linear dependence on $z / l$.

${ }^{18}$ For simplicity we consider the symmetric situation and drop the \pm signs in the superscripts.
} 
bation. From equation (57), the perturbation suffered by the $(+)$ wave packet upon traveling a distance $z \sim v_{\mathrm{A}} t$ is

$$
\delta \eta_{\lambda}^{+} \sim k_{\perp}^{2} \eta_{\lambda}^{+} \eta_{\lambda}^{+} \Delta \eta_{\lambda}^{-},
$$

where $\Delta \eta_{\lambda}^{-} \equiv \eta^{-}\left(\lambda_{\perp}, z\right)-\eta^{-}\left(\lambda_{\perp}, 0\right)$. Using $\eta_{\lambda} \sim l\left(v_{\lambda} / v_{\mathrm{A}}\right)$,

$$
\Delta \eta_{\lambda}^{-} \sim \frac{v_{\lambda}^{-}}{v_{\mathrm{A}}}(l z)^{1 / 2} .
$$

Thus the fractional distortion of the positively directed wave packet is

$$
\frac{\delta v_{\lambda}^{+}}{v_{\lambda}^{+}} \sim \frac{\delta \eta_{\lambda}^{+}}{\eta_{\lambda}^{+}} \sim \frac{l v_{\lambda}^{+}}{\lambda v_{\mathrm{A}}} \frac{l v_{\lambda}^{-}}{\lambda v_{\mathrm{A}}}\left(\frac{z}{l}\right)^{1 / 2},
$$

which is smaller than the corresponding quantity in intermediate turbulence (set $n=4$ in eq. [17], and compare with the above eq. [61]) by a factor $(z / l)^{1 / 2}$; thus, harmonic generation is unimportant for intermediate turbulence. ${ }^{19}$

\section{EVOLUTION OF A WEAK PERTURBATION}

Now we address the issue of the physical relevance of the cascade proposed by SG for weak turbulence. We go on to show that weak turbulence, intermediate turbulence, and strong turbulence can be consecutive stages of a single turbulent cascade.

Imagine exciting shear Alfvén waves (isotropically on scale $l / L \ll 1$ with amplitude $v_{l} / v_{\mathrm{A}} \ll 1$ ) in a cubical box filled with an electrically conducting fluid that is threaded by an unperturbed magnetic field aligned parallel to the $z$-axis. Let the box have side length $L$ and assume that its walls are made of an excellent electrical conductor. Then field-line displacements associated with turbulent motions must vanish at the walls. This boundary condition provides the cutoff of the energy spectrum for $k_{z} L<1$.

Suppose that

$$
\left(\frac{v_{l}}{v_{\mathrm{A}}}\right)^{2} \ll \frac{l}{L}
$$

Then field lines initially separated by scale $l$ maintain this approximate spacing. This ensures that there are no resonant three-wave interactions in the upper part of the cascade and that resonant four-wave interactions dominate. From equation (25), we have

$$
\frac{v_{\lambda}}{v_{\mathrm{A}}} \sim \frac{v_{l}}{v_{\mathrm{A}}}\left(\frac{\lambda}{l}\right)^{2 / 3} .
$$

The weak cascade grades into the intermediate cascade when

$$
\left(\frac{v_{\lambda}}{v_{\mathrm{A}}}\right)^{2} \sim \frac{\lambda^{2}}{l L}
$$

which occurs for

$$
\frac{\lambda_{1}}{l} \sim\left(\frac{v_{l}}{v_{\mathrm{A}}}\right)^{3}\left(\frac{L}{l}\right)^{3 / 2}
$$

\footnotetext{
${ }^{19}$ However, it might play an important role in strong turbulence.
}

For the intermediate cascade,

$$
\frac{v_{\lambda}}{v_{\mathrm{A}}} \sim\left(\frac{v_{l}}{v_{\mathrm{A}}}\right)^{3 / 2}\left(\frac{L}{l}\right)^{1 / 4}\left(\frac{\lambda}{l}\right)^{1 / 2} .
$$

The intermediate cascade steepens into the strong cascade when

$$
\frac{v_{\lambda}}{v_{\mathrm{A}}} \sim \frac{\lambda}{l}
$$

which takes place at

$$
\frac{\lambda_{2}}{l} \sim\left(\frac{v_{l}}{v_{\mathrm{A}}}\right)^{3}\left(\frac{L}{l}\right)^{1 / 2}
$$

Within the inertial range of the strong cascade,

$$
\frac{v_{\lambda}}{v_{\mathrm{A}}} \sim\left(\frac{v_{l}}{v_{\mathrm{A}}}\right)^{2}\left(\frac{L}{l}\right)^{1 / 3}\left(\frac{\lambda}{l}\right)^{1 / 3} .
$$

The complete three-dimensional inertial-range spectrum is given by

$$
\begin{aligned}
& E\left(k_{z}, k_{\perp}\right) \sim l^{3} v_{2}^{2}
\end{aligned}
$$

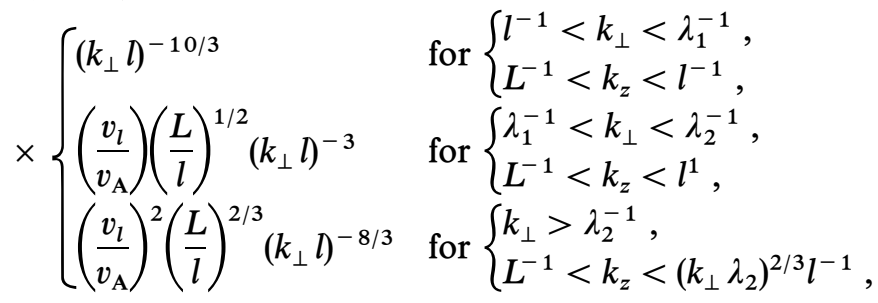

There are a couple of points worth noting in connection with the above combined cascade. The intermediate cascade is confined to $\lambda_{2} \lesssim \lambda \lesssim \lambda_{1}$, where the ratio

$$
\mathscr{R} \equiv \frac{\lambda_{2}}{\lambda_{1}} \sim \frac{l}{L}
$$

is independent of $v_{l} / v_{\mathrm{A}}$. As $\mathscr{R} \rightarrow 1$ from below, the inertial range of this cascade shrinks to zero, exposing a direct transition between the weak and strong cascades. This is the transition discussed in SG and Goldreich \& Sridhar (1995).

\section{DISCUSSION}

In a recent paper, $\mathrm{Ng} \&$ Bhattacharjee (1996) claimed that (1) in weak MHD turbulence, three-wave interactions between oppositely directed wave packets are nonzero if the $k_{z}=0$ components are nonzero and (2) three-wave interactions dominate over four-wave interactions. We hope to have persuaded the reader that (1) is correct so long as "weak" is altered to "intermediate" and that (2) is true only for individual collisions between small-amplitude wave packets. However, NB deserve full credit for demonstrating the importance of three-wave interactions, as well as discovering the frequency-changing terms in four-wave interactions. Both are a consequence of a nonzero net displacement of field lines, due to the perturbations of wave packets that have localized velocity and magnetic field perturbations.

Intermediate turbulence shares with weak turbulence the property that wave packets are long-lived: interaction times 
are much longer than the wave periods. The distinguishing feature is that perturbation theory is not applicable to intermediate turbulence; this should be clear from our demonstration that interactions of all orders have the same strength. Of course, during individual collisions, three-wave interactions dominate over all higher order interactions. However, as described in $\S 2.2 .2$, for wave packets localized in velocity and magnetic fields, but not in the net displacement of field lines, subsequent collisions are correlated; this makes all $n$-wave interactions contribute equally to intermediate turbulence. If Iroshnikov and Kraichnan had performed their heuristic estimates taking account of the fact that there is no parallel cascade for long-lived wave packets, they would have found the spectrum of the intermediate cascade. This is one case in which the assumption of isotropy (usually innocuous) is misleading; the anisotropic intermediate cascade strengthens on small spatial scales, whereas the isotropic IK cascade weakens.

We have devoted attention almost exclusively to shear Alfvén ("sA" for brevity) waves, ignoring the dynamics of pseudo-Alfvén ("pA") waves. ${ }^{20}$ A generic excitation may be expected to put power equally into both kinds of waves. Should not we then study the interactions of the pA waves among themselves, as well as with sA waves? Will this modify the cascades we derived for the sA waves? It turns out that the $\mathrm{pA}$ waves are slaved to the sA waves. The reason is as follows: Suppose that we were following the distortions suffered by a positively directed wave packet due to other, negatively directed ones. The nonlinear interactions are given, to order of magnitude, by some power of $\left(\boldsymbol{v}^{-} \cdot \nabla\right) v^{+} \sim\left(\boldsymbol{k} \cdot \boldsymbol{v}^{-}\right) \boldsymbol{v}^{+}$. Because the cascades in MHD turbulence are anisotropic, we expect that $k_{\perp} \gg k_{z}$ in the inertial range. We note that the polarization of an sA wave

\footnotetext{
${ }^{20}$ The only exception is the proof that there are no three-wave couplings for either type of Alfvén wave, in the Lagrangian perturbation theory of SG.
}

is essentially along $\hat{z} \times \boldsymbol{k}_{\perp}$, and that of a pA wave is along $\hat{z}$, and hence estimate that the "operator" $k \cdot v \sim\left(k_{\perp} v_{s} A\right)$ $+\left(k_{z} v_{p} A\right) \sim\left(k_{\perp} v_{s} A\right) .{ }^{21}$ Thus the $\mathrm{pA}$ waves are slaved to the sA waves; in a later paper, we will derive kinetic equations and demonstrate that the spectrum of the $\mathrm{pA}$ waves is identical to that of the sA waves for weak, intermediate, and strong turbulence.

How might an energy spectrum with a cutoff below $k_{z} L \sim 1$ arise? Two related possibilities come to mind. The first involves a thought experiment that could be realized computationally; we described this in $\S 4$. A more natural setting might be the atmosphere of a massive star that possesses a strong external dipole field. ${ }^{22}$ The energy spectrum of waves in the stellar magnetosphere would be cut off on scales longer than the length of the flux tubes that link the northern and southern magnetic hemispheres. The cutoff at small $k_{z}$ necessary for weak turbulence makes it, in general, less applicable than intermediate turbulence. However, the limited inertial ranges of both weak and intermediate cascades suggests that neither is likely to find much application in nature. The critically balanced cascade, proposed by Goldreich \& Sridhar (1995) for strong MHD turbulence, remains the most likely candidate for interstellar turbulence.

Financial support for this research was provided by NSF grant 94-14232. P. G. benefited from visits to the Institute for Advanced Study and the Canadian Institute for Theoretical Astrophysics. We thank Omer Blaes for comments that improved the clarity of the manuscript.

\footnotetext{
${ }^{21}$ If the amplitude of the pA wave is very much larger than the sA wave, we could imagine that $\left(k_{\perp} v_{s} A\right)<\left(k_{z} v_{p} A\right)$, but this is an unlikely situation. However, a detailed analysis rules out this possibility in cases in which both waves have comparable amplitude on the outer scale.

${ }^{22}$ Stars with masses in excess of a few solar masses have radiative atmospheres with little mass motion.
}

Frisch, U. 1995, Turbulence (Cambridge: Cambridge Univ. Press) Goldreich, P., \& Sridhar, S. 1995, ApJ, 438, 763

Iroshnikov, P. S. 1963, AZh, 40, 742

Kraichnan, R. H. 1965, Phys. Fluids, 8, 1385

Montgomery, D., \& Matthaeus, W. H. 1995, ApJ, 447, 706

Newcomb, W. A. 1962, Nucl. Fusion Suppl., 2, 451

Ng, C. S., \& Bhattacharjee, A. 1996, ApJ, 465, 845 (NB)

\section{REFERENCES}

Parker, E. N. 1979, Cosmical Magnetic Fields (Oxford: Oxford Univ. Press)

Shebalin, J. V., Matthaeus, W. H., \& Montgomery, D. 1983, J. Plasma Phys., 29, 525

Sridhar, S., \& Goldreich, P. 1994, ApJ, 432, 612 (SG)

Zakharov, V. E., L'vov, V. S., \& Falkovich, G. 1992, Kolmogorov Spectra of Turbulence, Vol. 1, Wave Turbulence (Berlin: Springer) 